\title{
A Narrative Review of the Laboratory Information System and Its Role in Antimicrobial Resistance Surveillance in South Africa
}

\author{
Peter S. Nyasulu1,2*, Christine Paszko3, Nontombi Mbelle ${ }^{4,5}$ \\ ${ }^{1}$ Department of Public Health, School of Health Sciences, Monash University, Johannesburg, South Africa \\ ${ }^{2}$ School of Public Health, Faculty of Health Sciences, University of the Witwatersrand, Johannesburg, \\ South Africa \\ ${ }^{3}$ Accelerated Technology Laboratories, Inc., West End, NC, USA \\ ${ }^{4}$ Department of Medical Microbiology, University of the Pretoria, Pretoria, South Africa \\ ${ }^{5}$ National Health Laboratory Services, Steve Biko Pretoria Academic Hospital, Pretoria, South Africa \\ Email: *peter.nyasulu@monash.edu
}

Received 11 June 2014; revised 12 July 2014; accepted 8 August 2014

Copyright (C) 2014 by authors and Scientific Research Publishing Inc.

This work is licensed under the Creative Commons Attribution International License (CC BY).

http://creativecommons.org/licenses/by/4.0/

(c) (i) Open Access

\begin{abstract}
A laboratory information system (LIS) established in a microbiology department has the potential to play an important role in the quality of microbiology data such as culture of blood, urine, stool, pus swab samples etc. Such data could be effectively utilised to measure the burden of antimicrobial resistance among patients presented to various hospitals and clinics with an episode of an infectious illness of bacterial origin. A variety of clinical and epidemiological investigations are conducted using culture data and the presence of an electronic system such as LIS enhances such investigations and improves the reliability of measures of antimicrobial resistance owing to improved data quality as well as completeness of data gathered as opposed to paper based system. Therefore to improve surveillance of antimicrobial resistance in South Africa, there is a need to reinforce the functionality of the LIS in both public and private microbiology laboratories as this will help to improve internal quality control methodologies.
\end{abstract}

\section{Keywords}

Laboratory Information Systems, Antimicrobial Resistance, Bacterial Pathogens, Surveillance, South Africa 


\section{Introduction}

The laboratory information system (LIS) is a data processing and dissemination technique used in the laboratory to deliver accurate and understandable results within a reasonable timescale as requested by clinicians. The system entails a sequence of events which include, delivery of samples to the laboratory, sample accessioning, analysing, verifying and approving results or reanalysing samples and releasing results to the clinicians who requested the tests [1]. In short, the concept of LIS refers to the computerisation of the laboratory system or automation of clerical labour-intensive activities associated with the processing of laboratory results to improve accuracy and turnaround time of results. Automation of laboratory activities removes the element of manual reporting, increases productivity and allows access to retrospective data for analysis [1]. Previous studies have reported an improvement in the accuracy of data and turnaround time of laboratory results after installation of the LIS [1].

The increase in clinical specimens as well as the consolidation and integration of laboratories and tests have resulted in the generation of significant volumes of tests and data. The complex and large quantity of data that these laboratories accumulate as well as the continued demand for data to support public health surveillance for effective disease prevention has resulted in the need for operational LIS or Laboratory Information Management System (LIMS). Although these terms are often used interchangeably, LIS typically refers to strictly clinical operations and LIMS refers to all others including Public Health, Pharmaceutical, Research and Development, Manufacturing, Food \& Beverage, Forensics, Chemicals and other fields as well. For the purpose of this paper we will use the term LIS. This system can efficiently integrate and handle all sophisticated processes and procedures related to data from different laboratory departments. These include microbiology, parasitology, virology, histopathology, biochemistry, hematology, endocrinology, cytology, toxicology, serology and immunology [1] [2]. Data for all such activities for laboratory services aggregates at the data warehouse repositories. This data is available to enhance business (i.e. billing of laboratory test), research and training aspects of the organisation.

\section{General Description of LIS Components and Function}

A laboratory information system is illustrated in Figure 1. The system is composed of the following components: hardware and network connectivity (computer system), LIS/LIMS software (computer programs), human capital (physicians who order laboratory tests, transport samples, etc.), trained laboratory staff who setup laboratory tests, execute procedures (i.e. blood culturing, HIV testing, susceptibility testing etc.) data analysis, quality control and reporting (laboratory results) (Figure 1).

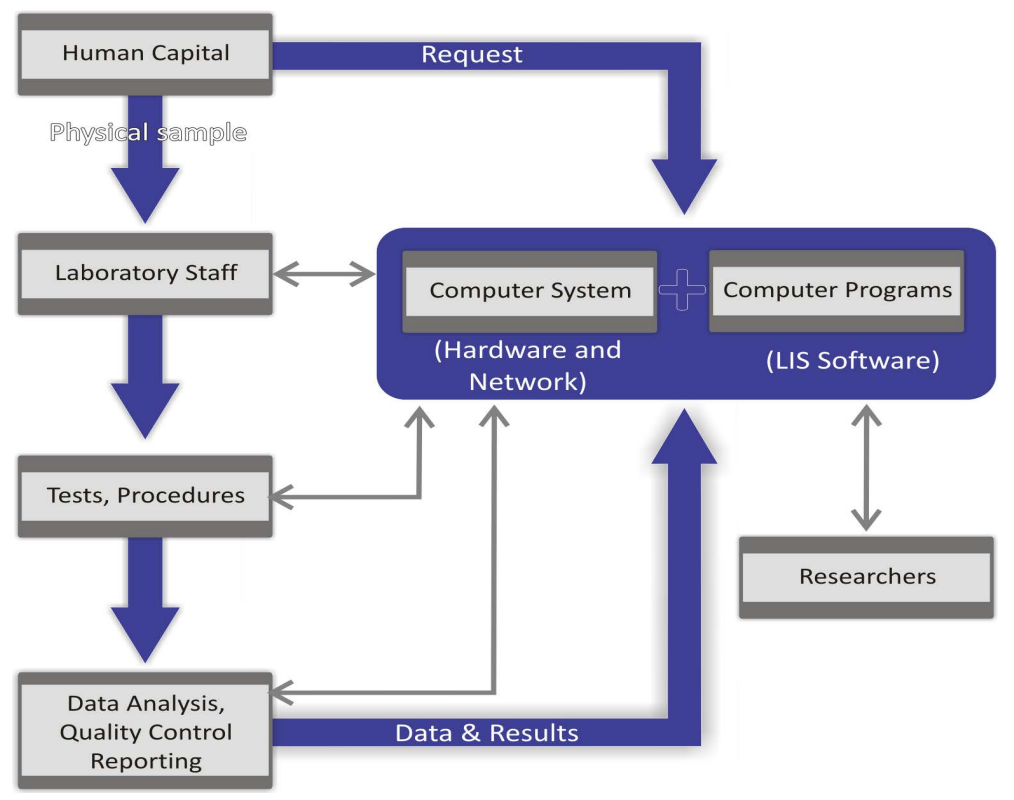

Figure 1. Flow chart demonstrating the components of a laboratory information management system. 
All components are integrated and support each other interactively in the collection, capture, processing, storage and the distribution of data obtained during analysis. The system puts the required efficiency of tracking and sorting laboratory data, improves turnaround time of laboratory results and allows for the retrospective analysis of data for surveillance or research purposes [3]. Figure 2 describes the processes and procedures in the function of a LIS [4].

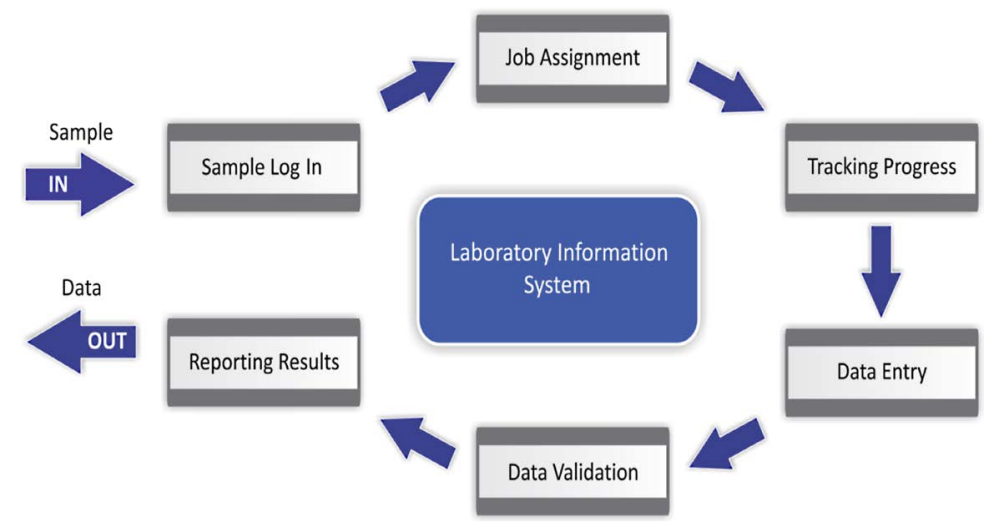

Figure 2. Laboratory information management system processes and procedures [4]. Adapted from Zhu, J. Indiana University, 2005.

http://hdl.handle.net/1805/323

Although the LIS is a complex system, it works to simplify and improve the efficiency of laboratory operations, minimise data entry and other transcription errors as well as deliver valid and reliable laboratory results to the client in the most efficient way. In addition, the system provides a platform for surveillance such as monitoring antimicrobial resistance following the utilization of retrospective data archived in the LIS database [3] [5]. In South Africa, for example, the National Health Laboratory Services (NHLS) [2] a network of public health laboratories with a national footprint utilizes LIS software known as DISAlab that was supplied and maintained by the Laboratory System Technologies (Pty) Limited for the past two decades. However the DISA LIS has been phased out and replaced by the TrakCare LIS software developed by the Health Systems Technology [5] [6]. The LIS as a way of internal quality control measures allows for validation of data entries to detect errors as the system has built in check codes. In the event of failure, verification of data entered is then done manually by an alternative laboratory staff member. In addition, an external quality control process exists in microbiology laboratories. The accreditation or quality assurance process of each laboratory allows for improvement in the quality and reliability of data produced [7]. An acceptable margin of error of 5\% indicating observational error in reporting quantitative measures should be taken into account when analysing routine microbiology data due to missing data which is an inherent weakness of the routine passive surveillance system. Some of the parameters entered into the LIS database are the following: Sample identification, time collected, time registered; demographics such as date of birth or estimated age, gender, hospital, ward, province, clinical diagnosis (scanty data),organism cultured, drug sensitivity (resistant/sensitive (R/S); minimum inhibitory concentration (MIC)), date sample was first registered and tested, date and time results were reviewed, instrument used for testing, sensitivity of antimicrobials, date clinician printed the results as well as first laboratory where specimen was taken. In South Africa, data from microbiology laboratories are routed to a central server the corporate data warehouse (CDW) [8] a data repository that is interlinked to the LIS. This includes data from cultures of blood, cerebral spinal fluid, stool and urine, pus swabs among others. Such routinely collected data could be used to monitor patterns and trends of antimicrobial resistance. The CDW composition [8] however provides limited platform for epidemiological investigations that aims to investigate specific clinical outcomes associated with antimicrobial resistance. The reason being that laboratory data originating from blood, urine, pus swabs etc., does not often contain clinical parameters such as severity of illness, outcome of treatment etc. This would henceforth make investigations linking clinical outcomes to antimicrobial resistance uncertain to undertake.

\section{Variation in LIS}

There are major challenges relating to the NHLS LIS which might originate from wide variation in the opera- 
tions of the LIS between different laboratories. These emanate from wide variations in procedures that are used for gathering and reporting of culture and sensitivity data. Some of the underlying causes of such wide variability might be: 1) different reporting styles between different laboratories including different names used by different instruments; 2) instruments used vary between different laboratories i.e. other laboratories use more advanced instruments than others; 3) lack of standardisation across different laboratories which might affect scope of data generated.

\section{Data Security System}

The LIS data is password protected and each of the local laboratories has an electronic gate keeper, to monitor and minimise data corruption, and to access the data for research use (https://labresults.nhls.ac.za/). Since data originates from varying sources, the potential for data corruption is high hence appropriate security measures for access and utilisation are essential.

\section{Data Quality}

For an effective surveillance system to be sustained, data quality (i.e. completeness of demographics e.g. age, gender, geographical location; antibiotic susceptibility tests (i.e. MIC), resistant/sensitivity etc.) needs to have a primary focus. For this reason, it is paramount to monitor specific areas of the system where problems with data quality could be identified and appropriate intervention undertaken to improve data quality. To achieve this, the following issues need to be considered: 1) whether a researcher would accept at face value what was extracted from the database using the designed query; and 2) whether a researcher would be able to make any request for specific, logical, clear and unambiguous data elements in the query design. To improve the quality of data aggregated at the CDW [8], there is need for various players from the different microbiology laboratories to work collaboratively so as to minimise major variability of antimicrobial resistance patterns that might be originating to a large extent from data entry errors. Regular quality control exercises are essential to improve accuracy and reliability of culture data. To improve surveillance of antimicrobial resistance, in public sector hospitals the NHLS should introduce instruments that could be utilized effectively to generate reliable data elements to be used for various epidemiological investigations as well as aid clinical decision making regarding bacteraemia episode by clinicians in the clinical departments [9].

\section{Conclusion}

The LIS was not primarily designed as a research or surveillance tool. Its function has been to generate data accessible to the requesting client that could be used for appropriate and accurate billing of all tests done in the laboratory. Data has also been used to understand the volume of tests done, the time it takes to get results back to the patient and to plan efficient service delivery of the NHLS. We believe that such a system can be used as an effective surveillance tool to monitor development of antimicrobial resistance to various significant bacterial pathogens in our population since the process of acquiring culture and sensitivity data is inherently ongoing. Therefore, understanding the challenges of the system and suggesting ways of improving the overall system performance would be a step in the right direction for a well-established and functioning antimicrobial resistance surveillance program. Such a program would enhance our ability to contain the growing crisis of antimicrobial resistance that threaten our ability to treat patients effectively in South Africa.

\section{Acknowledgments}

We would like to acknowledge Prof Jill Murray for her critical comments and editorial contribution to this manuscript.

\section{References}

[1] Paszko, C. (2014) Computerised Laboratory Information Management System (LIMS). www.samedanltd.com

[2] NHLS (National Health Laboratory Services) (2013) http://www.nhls.ac.za

[3] Skolbelev, D.O., Zaytseva, T.M., Kozlov, A.D., Perepelitsa, V.L. and Makarova, A.S. (2011) The Metrological Service: Labaratory Information Management Systems in the Work of the Analytic Laboratory. Measurement Techniques, 53, 
1182-1189. http://dx.doi.org/10.1007/s11018-011-9638-7

[4] Zhu, J.Y. (2005) Automating Laboratory Operations by Intergrating Labortory Information Management Systems (LIMS) with Analytical Instruments and Scientific Data Mangement Systems (SDMS). Indiana University. http://hdl.handle.net/1805/323

[5] Health Systems Technologies. Laboratory Information System. http://www.healthsystems.co.za/?page_id=19

[6] Patil, P.S., Rao, S. and Patil, S.B. (2011) Optimization of Data Warehousing System: Simplification in Reporting and Analysis. IJCA Proceedings on International Conference and Workshop on Emerging Trends in Technology (ICWET), 9, 33-37. http://www.ijcaonline.org/proceedings/icwet/number9/2131-db195

[7] TrakCare Lab. Breakthroughs in Patient Outcomes, Lab Performance and Clinicians Communication. http://www.intersystems.com/TrakCareLAB/TrakCareLAB.pdf

[8] Hirsh, J. (1999) Open Database Connectivity Version 08. www.erlang.org/documentation/doc-4.9.1/pdf/odbc-0.8.1.pdf

[9] Haynes, R.B. and Wilczynski, N.L. (2010) Effects of Computerized Clinical Decision Support Systems on Practitioner Performance and Patient Outcomes: Methods of a Decision-Maker-Researcher Partnership Systematic Review. Implementation Science, 5, 12. http://www.implementationscience.com/content/5/1/12 http://dx.doi.org/10.1186/1748-5908-5-12 
Scientific Research Publishing (SCIRP) is one of the largest Open Access journal publishers. It is currently publishing more than 200 open access, online, peer-reviewed journals covering a wide range of academic disciplines. SCIRP serves the worldwide academic communities and contributes to the progress and application of science with its publication.

Other selected journals from SCIRP are listed as below. Submit your manuscript to us via either submit@scirp.org or Online Submission Portal.
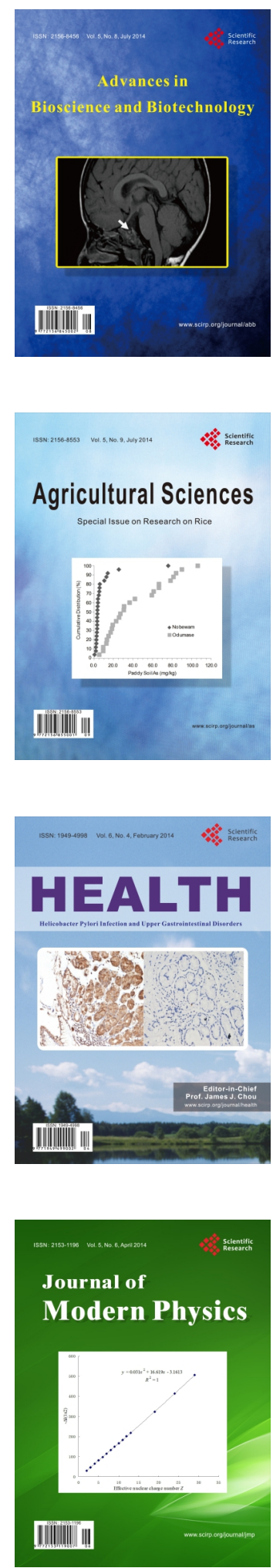
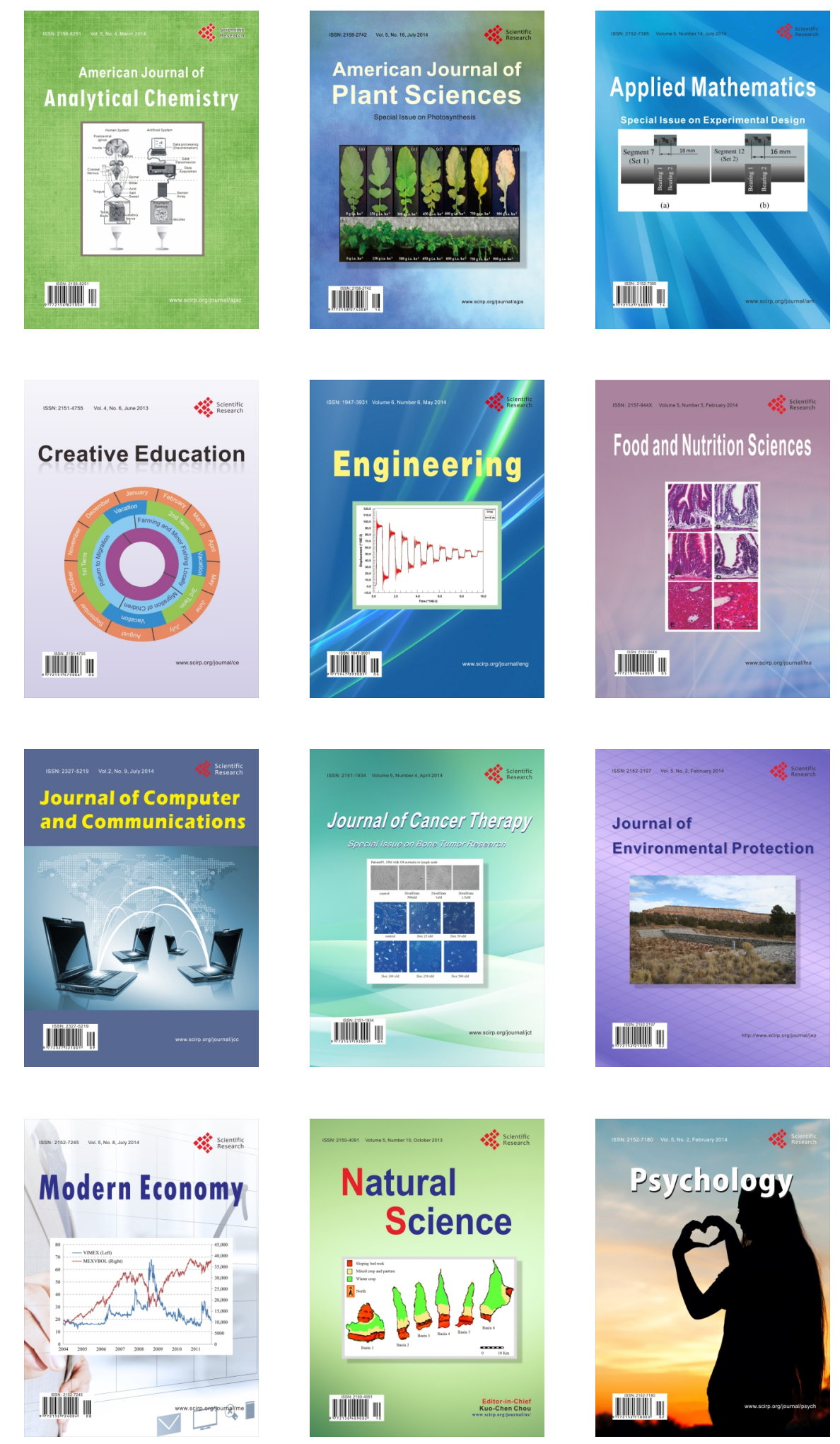